\title{
Remarks on Duality of 1 Dimensional Quantum Spin Models
}

\author{
Taku Matsui \\ Department of Mathematics, Tokyo Metropolitan University, 1-1 Minami Ohsawa, Hachioji-shi, \\ Tokyo, Japan
}

Received January 21, 1992; in revised form May 15, 1992

\begin{abstract}
We present some results on duality maps and ground states of 1 dimensional quantum spin models. We also give some applications to Kramers Wannier duality and the nonlocal transformation that Kennedy and Tasaki discovered in their study of Haldane phase of quantum antiferromagnetic spin models.
\end{abstract}

\section{Introduction}

In this note, we consider certain nonlocal transformations which are used in the study of 1 dimensional quantum spin models. Examples are Kramers Wannier duality for quantum Potts models and the nonlocal transformation of Kennedy and Tasaki discovered in their study of Haldane phase of quantum antiferromagnetic spin models [5]. We will consider infinite volume ground states determined by these nonlocal transformations.

The ground state structure of quantum antiferromagnetic spin models attracted much attention of solid state physicists recently. Even in the 1 dimensional case, the characterization of phases is not yet fully understood. The isotropic antiferromagnetic Heisenberg model is believed to have a unique massless infinite volume ground state in the half odd integer spin case while Haldane's conjecture states that the model is massive in the integer spin (see [2]). However, it seems that no rigorous proof for uniqueness of the infinite volume ground state exists even in the spin 1/2 Heisenberg model which is a typical "solvable" model in the sense of Baxter ([2] deals with only the infinite volume limit under the free boundary condition while we expect that the infinite volume limit in any boundary condition converges to the same infinite volume state.) We believe that the rigorous study of infinite volume ground states is not merely of mathematical interest.

One nice feature of the 1 dimensional quantum spin model is existence of nonlocal transformations (e.g. bosonization, Kramers Wannier duality, etc.) that make some models formally equivalent to other simpler models. Such formal transformations give rise to "dual fields" (alternatively called disorder variables). The aim of this note 
is to present results on duality transformations of quantum spin models in the infinite volume ground states. We consider the relation between ground states of original and transformed systems.

The duality techniques are old (see [6] and the references therein). However, mathematically speaking, the existence of dual fields on the physical Hilbert space is quite subtle. In the case of the 1 dimensional $X Y$ model, we can prove rigorously nonexistence of dual variables on the ground state representation when the Hamiltonian has no mass gap in its spectrum by use of ideas of [1]. The results of this note are distinguished from other results ([6] for example) in the following points. (a) We do not assume the existence of dual fields and we carry out our proof, looking only at the globally gauge invariant observables on which the duality transform is well defined. (b) We consider quantum spin models. The most important example of this paper is the deformation of the AKLT model (see below and Theorem 4.1). Hence Lorentz or spatial rotation invariance is not available and as a consequence cluster properties of correlation functions at large spatial distance have nothing to do with spectral gap of Hamiltonian.

We are going to use the $C^{*}$ algebraic method for study of infinite volume quantum models. The basic reference of this approach is [4].

We consider 1 dimensional spin models and the algebra of quantum observables $A$ is the infinite tensor product of matrix algebra,

$$
A=\bar{\bigotimes}_{Z} M_{n}(C){ }^{C^{*} \text { algebra }},
$$

where $M_{n}(C)$ is the set of all $n$ by $n$ matrices and by $-C^{*}$ algebra we denote the $C^{*}$ algebra completion.

Let $Q$ be a $n$ by $n$ matrix. Then by $Q^{(j)}$ we denote the observable $Q$ on the site $j$. We will also use the lattice translation automorphism $\alpha_{k}(\cdot)$ ( $k$ is an integer) determined by

$$
\alpha_{k}\left(Q^{(j)}\right)=Q^{(j+k)},
$$

$A_{\text {loc }}$ is the dense subalgebra of strictly local elements generated by $\left\{a^{(j)}\right\}$.

The Hamiltonian is usually a (formal) sum of selfadjoint strictly local elements and in this note we consider translationally invariant or periodic Hamiltonians,

$$
H=\sum_{k \in \mathrm{p} Z} \alpha_{k}(h),
$$

where $p$ is the period and $h=h^{*} \in A_{\mathrm{loc}}$.

The nonlocal transformations mentioned above are described as follows.

(i) Kramers Wannier duality for quantum Potts models. We consider $n$ state quantum Potts models. The Hamiltonian is the time continuum limit of the logarithm of the transfer matrix for the 2 dimensional Potts model.

Let $U$ and $V$ be $n$ by $n$ matrices defined by

$$
U=\left[\begin{array}{ccccc}
1 & 0 & \cdots & \cdots & 0 \\
0 & \omega & 0 & \cdots & 0 \\
0 & 0 & \omega^{2} & 0 & 0 \\
0 & \cdots & 0 & \cdots & 0 \\
0 & \cdots & \cdots & 0 & \omega^{n-1}
\end{array}\right], \quad V=\left[\begin{array}{cccc}
01 & 0 & \cdots & 0 \\
00 & 10 & \cdots & \\
\ldots \ldots & \ldots & \ldots & \ldots \\
0 & \cdots & \cdots & 01 \\
10 & 0 & \cdots & 00
\end{array}\right],
$$


where $\omega$ is the $n^{\text {th }}$ primitive root of 1 .

The matrices $U$ and $V$ are the $n$ state analogue of Pauli spin matrices $\sigma_{x} \sigma_{z}$. They satisfy the following relations:

$$
\begin{gathered}
U^{n}=U U^{*}=U^{*} U=V^{n}=V V^{*}=V^{*} V=1, \\
U V=\omega^{n-1} V U .
\end{gathered}
$$

Then we consider $U^{(j)}$ and $V^{(j)}$ in $A_{\text {loc }}$ and we set

$$
W_{j}= \begin{cases}V^{(j)} & \text { if } j \text { is an integer } \\ U^{(\jmath-1 / 2)}\left(U^{(j+1 / 2)}\right)^{*} & \text { if } j \text { is a half odd integer }\end{cases}
$$

The following identity can be easily verified.

$$
\begin{gathered}
W_{j} \cdot W_{j+1 / 2}=\omega W_{j+1 / 2} \cdot W_{j} \\
W_{j} \cdot W_{k}=W_{k} \cdot W_{j} \quad \text { if }|k-j| \geq 1
\end{gathered}
$$

Let $\beta($ ) be the automorphism determined by

$$
\beta(Q)=\lim _{M \rightarrow \infty} \prod_{|j| \leq M} V^{(j)} Q \prod_{|j| \leq M} V^{(j)-1} .
$$

Then $\beta\left(\right.$ ) induces an action of the group $\mathbf{Z}_{\mathbf{n}}=\mathbf{Z} / \mathbf{n} \mathbf{Z}$ because $\beta()^{n}=$ identity.

We consider the fixed point algebra of this action $A^{Z_{n}}$

$$
A^{Z_{n}}=\{Q \in A \quad \beta(Q)=Q\}
$$

It is not difficult to show that $\left\{W_{\jmath}: j\right.$ half integer $\}$ generates $A^{Z_{n}}$.

The Kramers Wannier duality map is an automorphism of $A^{Z_{n}}$ determined by the following equation:

$$
\tau_{K W}\left(W_{j}\right)=W_{j+1 / 2}
$$

We can also check

(i) $\tau_{K W}^{2}=\alpha_{1}, \tau_{K W} \circ \alpha_{k}=\alpha_{k} \circ \tau_{K W}$,

(ii) $\tau_{K W}$ cannot be extended to an automorphism of $A$.

The Hamiltonian of the quantum Potts model is

$$
H(\lambda)=-\sum_{j \in Z}\left\{e_{\jmath}+\lambda e_{j+1 / 2}\right\},
$$

where $\lambda$ is a real parameter and

$$
e_{j}=\frac{1}{n}\left(\sum_{l=0}^{n-1}\left(W_{j}\right)^{l}\right)
$$

This model is selfdual in the sense that

$$
\tau_{K W}(H(\lambda))=\lambda H(1 / \lambda)
$$


If $|\lambda|$ is small, the model has a unique translationally invariant ground state.

The correlation function is represented by a Gibbs measure of 1 dimensional classical spin system. See [8].

(ii) Duality of Kenneda and Tasaki for spin 1 model [5]. In their study of the Haldane phase, Kennedy and Tasaki discovered the following unitary which transforms the AKLT Heisenberg Hamiltonian [3] into a model having product states as its ground state. The transformation of Kennedy and Tasaki is highly nonlocal and breaks the $S U(2)$ symmetry but the Hamiltonian after the transformation admits a convergent polymer expansion for certain perturbation. Unfortunately not all the expected properties of Haldane phase were proved in [5]. Our results imply uniqueness of ground state and existence of mass gap (see Sect.4).

We now introduce the duality transformation of [5]. We consider the spin 1 case $(n=3)$. Let $S_{\alpha}^{(j)}(\alpha=x, y, z)$ be the spin 1 operator on the site $j$.

The AKLT Hamiltonian $H_{\mathrm{AKLT}}$ is the $S O(3)$ invariant model with the Hamiltonian,

$$
H_{\mathrm{AKLT}}=\sum_{j \in Z}\left\{\left(S^{(\jmath)} \cdot S^{(\jmath+1)}+\frac{1}{3}\left(S^{(j)} \cdot S^{(j+1)}\right)^{2}\right\} .\right.
$$

The exact ground state is known. It is a unique translationally invariant ground state with exponential decay of correlation and mass gap.

The duality transformation is now associated to the following $\mathbf{Z}_{\mathbf{2}} \times \mathbf{Z}_{\mathbf{2}}$ symmetry.

The elements of the group $\mathbf{Z}_{2} \times \mathbf{Z}_{2}$ are denoted by,,+++--+ and -- and $\beta_{x y}(x, y=+$ or -$)$ is an automorphism of the $C^{*}$ algebra $A$ determined via the formulae, $\beta_{++}=$identity and,

$$
\begin{gathered}
\beta_{+-}\left(S_{x}^{(j)}\right)=-S_{x}^{(j)}, \quad \beta_{+-}\left(S_{y}^{(j)}\right)=-S_{y}^{(j)}, \quad \beta_{+-}\left(S_{z}^{(j)}\right)=S_{z}^{(j)} \\
\beta_{-+}\left(S_{x}^{(j)}\right)=S_{x}^{(j)}, \quad \beta_{-+}\left(S_{y}^{(j)}\right)=-S_{y}^{(j)}, \quad \beta_{-+}\left(S_{z}^{(j)}\right)=-S_{z}^{(j)} \\
\beta_{--}=\beta_{-+} \circ \beta_{+-}=\beta_{+-} \circ \beta_{-+} .
\end{gathered}
$$

The fixed point algebra by this action (1.14) is denoted by $A^{Z_{2} \times Z_{2}}$.

$$
A^{Z_{2} \times Z_{2}}=\left\{Q \in A \quad \beta_{x y}(Q)=Q \quad x, y= \pm\right\} .
$$

Let $m$ be a positive integer and consider the following selfadjoint unitary $V_{m}$ :

$$
V_{m}=\prod_{k=-m}^{m} Y_{k}
$$

where

$$
\begin{aligned}
& Y_{k}=\left\{E_{k} \exp \left(\sqrt{-1} \pi S_{x}^{(k)}\right)+\left(1-E_{k}\right)\right\} \\
& E_{k}=\frac{1}{2}\left[1-\exp \left(\sqrt{-1} \pi \sum_{l=-m}^{k-1} S_{z}^{(l)}\right)\right]
\end{aligned}
$$

Then set

$$
\lim _{m \rightarrow \infty} V_{m} H_{\mathrm{AKLT}} V_{m}^{-1}=\underline{H} .
$$


Even though the transformation is nonlocal, the Hamiltonian $\underline{H}$ is of finite range.

We have 4 to 1 correspondence of infinite volume ground states of $H_{\mathrm{AKLT}}$ and $\underline{H}$ (see [5]). We will try to find the condition for the appearance of this 4 to 1 correspondence in Sect. 3 .

In the present situation, the duality map $\tau_{K T}$ we will consider is

$$
\tau_{K T}(Q)=\lim _{m \rightarrow \infty} V_{m} Q V_{m}^{-1}
$$

The limit (1.17) exists iff $Q$ is $A^{Z_{2} \times Z_{2}}$. We can also show that (i) $\tau_{K T}^{2}=$ identity, (ii) $\tau_{K T}$ cannot be extended to $A$.

We are going to consider these maps in one framework in Sect. 2. In Sect. 3 we give necessary and sufficient conditions for purity of states transformed by the duality map of Kennedy and Tasaki in the presence of string order. In Sect. 4 we explain the implication of results of Sect. 2 to the deformation of the AKLT model.

\section{Infinite Volume Ground States and Mass Gap}

In this section, we give some results on ground states after the duality transformation. We suppose that $G$ is a finite abelian group and $A$ is the $C^{*}$ algebra of observables.

$$
A=\bigotimes_{Z} M_{n}(C){ }^{C^{*} \text { algebra }}
$$

$G$ is a (global) gauge group of $A$, so $G$ acts on $A$ via the automorphism $\beta_{g}$ (). By $A^{G}$ we denote the fixed point algebra of this action $\beta_{g}($ ),

$$
A^{G}=\left\{Q \in A \quad \beta_{g}(Q)=Q \text { for any } g \in G\right\} .
$$

$\alpha_{k}(\cdot)(k \in Z)$ is the (lattice) translation defined in (1.2). We also assume that $\beta_{g}()$ commutes with $\alpha_{p}(\cdot)$ where $p$ is the period.

$$
\alpha_{p} \circ \beta_{g}(\cdot)=\beta_{g} \circ \alpha_{p}(\cdot)
$$

We will consider periodic Hamiltonians with finite range interaction,

$$
H=\sum_{l \in p Z} \alpha_{l}(h)
$$

where $p$ is the period of the Hamiltonian $H$.

We assume that $H$ is selfadjoint, of finite range and $G$ invariant, namely

$$
h=h^{*} \in A_{\mathrm{loc}} \cap A^{G} .
$$

Duality map $\tau(\cdot)$ is an automorphism of $A^{G}$ satisfying the following properties:

(i) $\tau(\cdot)$ cannot be extended to $A$.

(ii) $\tau(\cdot)$ maps any strictly local element of $A^{G}$ to a strictly local element of $A^{G}$. 
(iii) $\tau(\cdot)$ commutes with $\alpha_{p}(\cdot)$ for the period $p$.

$$
\alpha_{p} \circ \tau(Q)=\tau \circ \alpha_{p}(Q)\left(Q \in A^{G}\right) .
$$

The Hamiltonian determines the time evolution of an observable $Q$ via the formula,

$$
\gamma_{t}(Q)=e^{i t H} Q e^{-i t H}(Q \in A)
$$

By our assumption (2.5), $\gamma_{t}$ commutes with $\alpha_{p}(\cdot)$ and $\beta_{g}()$. by

We also consider the dual hamiltonian $H_{\tau}$ and the time evolution $\gamma_{t}^{\tau}$ determined

$$
\begin{gathered}
H_{\tau}=\tau(H)=\sum_{l \in p Z} \tau \circ \alpha_{l}(h), \\
\gamma_{t}^{\tau}(Q)=e^{i t H_{\tau}} Q e^{-i t H_{\tau}}(Q \in A) .
\end{gathered}
$$

Next we recall the standard definition of infinite volume ground states.

Let $\varphi$ be a state of $A$. $\varphi$ is a ground state of $H$ iff

$$
\left.\frac{1}{i} \frac{d}{d t} \varphi\left(Q^{*} \gamma_{t}(Q)\right)\right|_{t=0} \geq 0
$$

for any $Q$ in $A$ which is differentiable with respect to $\gamma_{t}$.

Let $\varphi$ be a periodic state with period $q$. We assume that $q$ is an integer multiple of $p$. Then $\varphi$ is a $q$ periodic ground state if and only if

$$
\varphi\left(\sum_{k=0}^{q-1} \alpha_{k}(h)\right)=\inf \left\{\psi\left(\sum_{k=0}^{q-1} \alpha_{k}(h)\right): \psi q \text { periodic state }\right\}
$$

See [4]. (2.9) implies the following lemma (cf. [9]).

Lemma 2.1. Take a selfadjoint local observable $Q=Q^{*}$ in $A_{\mathrm{loc}}$ and set

$$
H(\delta)=\sum_{j \in p Z}\left\{\alpha_{j}(h)+\delta \alpha_{j}(Q)\right\}=H+\delta \sum_{j \in p Z} \alpha_{j}(Q)
$$

where $\delta$ is a small real parameter. Let $\psi$ be a q periodic ground state of $H(\delta)$ and

$$
e(\delta)=\sum_{j=0}^{q-1} \psi\left(\alpha_{j}(h)+\delta \alpha_{j}(Q)\right)
$$

Suppose that $e(\delta)$ is differentiable (as a function of $\delta$ ) at $\delta=0$. Then for any $q$ periodic ground state $\varphi$ of $H$, we have

$$
\varphi(Q)=\left.\frac{d}{d \delta} e(\delta)\right|_{\delta=0}
$$


Remark 2.2. This is a corollary of a theorem of Brattelli, Kishimoto and Robinson. (See 6.2.58 in [4].) Lemma 2.1 is useful for showing uniqueness of the translationally invariant ground state. If we are able to prove differentiability of $e(\delta)$ for any selfadjoint $Q$ in $A^{G}$, the above lemma tells us the uniqueness of the $q$ periodic $G$ invariant ground state of $H$. For certain simple Hamiltonians, this program can be carried out. See [5].

Next we consider mass gap of $H$ and $H_{\tau}$. In the infinite volume case, the Hamiltonian $H$ does not make sense in itself as it is a sum of infinitely many element of $A$. The meaningful Hamiltonian is defined on the physical Hilbert space. If we have a ground state $\varphi$ of $H$, we consider the GNS representation of $A$ and the effective Hamiltonian on this Hilbert space is a selfadjoint positive operator which gives rise to the same time evolution $\gamma_{t}$. More precisely, let $F_{\varphi}$ be the GNS Hilbert space, $\Omega_{\varphi}$ be the GNS cyclic vector in $F_{\varphi}$,

$$
\varphi(Q)=\left(\Omega_{\varphi}, Q \Omega_{\varphi}\right)
$$

we have a selfadjoint operator $H_{\varphi}$ on $F_{\varphi}$ satisfying

$$
H_{\varphi} \geq 0, \quad H_{\varphi} \Omega_{\varphi}=0
$$

and

$$
e^{i t H_{\varphi}} Q e^{-i t H_{\varphi}}=\gamma_{t}(Q) \text { for any } Q \text { in } A
$$

The following results are known.

(i) $H_{\varphi}$ is uniquely determined by these equations.

(ii) $H_{\varphi}^{\varphi}$ is affiliated with the von Neumann algebra generated by $A$ in $F_{\varphi}$,

$$
e^{i t H_{\varphi}} \in A^{\prime \prime}
$$

(iii) $\varphi$ is pure (the GNS representation is irreducible) if the multiplicity of zero eigenvalue is one (cf. Proposition 5.3.19 of [4]).

Definition 2.3. $H_{\varphi}$ (or the state $\varphi$ ) has mass gap if and only if there exists a positive constant $m$ such that

$$
\text { Spectrum } H_{\varphi} \cap(0, m)=\phi
$$

We first state the relation between ground state of $H$ of (2.3) and $H_{\tau}$ of (2.6). Let $E^{G}$ ( ) be the projection from $A$ to $A^{G}$ defined by

$$
E^{G}(Q)=\int_{G} d g \alpha_{g}(Q)
$$

where $Q$ is in $A$ and $d g$ is the normalized Haar measure (a finite sum in our case). 
A $q$ periodic state $\varphi$ of the $C^{*}$ algebra $A$ is mixing if for any $Q_{1}$ and $Q_{2}$ in $A$,

$$
\lim _{|n| \rightarrow \infty} \varphi\left(Q_{1} \alpha_{n q}\left(Q_{2}\right)\right)=\varphi\left(Q_{1}\right) \varphi\left(Q_{2}\right)
$$

Proposition 2.4. (i) Let $\psi$ be a q periodic ground state of $H_{\tau}$. Let $\varphi$ be a state of $A$ defined by

$$
\varphi(Q)=\psi \circ \tau \circ E^{G}(Q),
$$

then $\varphi$ is a q periodic $G$ invariant ground state of $H$.

(ii) (2.17) gives a 1 to 1 correspondence between the set of $q$ periodic $G$ invariant ground states of $H$ and those of $H_{\tau}$. In particular $H$ has a unique q periodic ground state if $H_{\tau}$ has a unique $q$ periodic and $G$ invariant ground state $\psi()$ and the state $\varphi$ defined by (2.17) is mixing.

Remark 2.5. We remark that if one wants to show the exponential decay of correlation of $\varphi$ one must show the exponential decay of correlation $\psi$ for $G$ invariant observables in $A^{G}$ and exponentially fast convergence of

$$
\lim _{|k| \rightarrow \infty} \psi \circ \tau\left(Q_{1} \alpha_{k}\left(Q_{2}\right)\right)=0
$$

where $Q_{1} Q_{2}$ is in $a^{G}$ and for a non-trivial irreducible representation $\pi($ ) of $G$,

$$
\beta_{g}\left(Q_{1}\right)=\pi(g) Q_{1}
$$

Note that $Q_{1} \alpha_{k}\left(Q_{2}\right)$ can be highly non-local.

Proposition 2.6. Let $\psi()$ be a periodic ground state of $H_{\tau}$ and $\varphi()$ be the ground state of $H$ determined by (2.17). Suppose that $\varphi()$ is mixing. Then, $\varphi()$ has mass gap if and only if (2.15) is valid for $\psi()$.

Remark 2.7. Proposition 2.4 may not be valid for non-translationally invariant ground states. A counterexample is the quantum Ising model $(n=2$ in the example (1.11)). If $\lambda=0$ the ground state is unique while the $\lambda=\infty$ case has non-translationally invariant ground states. See [1].

For certain models (models of [7] and the AKLT model (1.13)) we can also consider another definition of ground state. A state $\varphi$ may be called a ground state if it satisfies the following equation for any integer $n$ :

$$
\begin{aligned}
\varphi\left(\sum_{k=n}^{q+n} \alpha_{k}(h)\right) & =\inf \left\{\psi\left(\sum_{k=0}^{q-1} \alpha_{k}(h)\right): \psi \text { state }\right\} \\
& =\inf \text { spectrum of } \sum_{k=0}^{q-1} \alpha_{k}(h) .
\end{aligned}
$$

A state satisfying (2.19) may not exist. If it exists, it is a ground state in the sense of (2.8). Examples of non-translationally invariant ground state satisfying (2.19) can be constructed by the technique of [7]. Results stated in Propositions 2.4 and 2.6 are valid if we replace $q$ periodic ground states by ground states satisfying (2.19). 
Proof of Proposition 2.4. (i) follows from our assumption (2.5) and (2.9). In fact, by $G$ invariance of $h$,

$$
\varphi(h)=\psi \circ \tau \circ E^{G}(h)=\psi(\tau(h)) .
$$

The right-hand side of (2.9) is independent of the choice of a $q$ periodic state $\varphi()$ and we may call the right-hand side of (2.9) the ground state energy of $h$. The inf can be taken in $G$ invariant $q$ periodic states in (2.9). As $\tau$ is an automorphism of $A^{G}$, the ground state energy of $h$ and $\tau(h)$ are same.

The first statement of (ii) is obvious. The second statement follows from the fact that any $q$ periodic mixing state is extremal among $q$ periodic states (see Theorem 4.3.17 of [4]). If $\omega$ is a $q$ periodic ground state, $\omega \circ E^{G}=\varphi$ so we have $\omega=\varphi$.

Proof of Proposition 2.6. Let $F_{\varphi}$ (respectively $F_{\psi}$ ) be the GNS representation space of $\varphi$ (respectively $\psi$ ) and $\Omega_{\varphi}$ (respectively $\Omega_{\psi}$ ) be the associated cyclic vector. By $G$ invariance and periodicity of states, there exist the unitary operators $V_{g}$ and $W$ implementing automorphisms $\beta_{g}(g$ in $G)$ and $\alpha_{q}$.

$$
\begin{aligned}
& W Q W^{-1}=\alpha_{q}(Q), \quad W \Omega_{\varphi}=\Omega_{\varphi} \\
& V_{g} Q V_{g}^{-1}=\beta_{g}(Q), \quad V_{g} \Omega_{\varphi}=\Omega_{\varphi} .
\end{aligned}
$$

Furthermore $V_{g}$ is a representation of $G$ and the Hilbert space $F_{\varphi}$ is decomposed into $A^{G}$ invariant subspaces $F_{\chi}$ labeled by irreducible representations $\chi$ of the (finite abelian) gauge group $G$,

$$
F_{\varphi}=\sum_{\chi \in \hat{G}}^{\oplus} F_{\chi} .
$$

We call each $F_{\chi}$ a sector following the convention of quantum field theory. By $F_{0}$ we denote the sector for the trivial representation and we call it the vacuum sector. $\Omega_{\varphi}$ belongs to $F_{0}$. By the $G$ invariance of state the operator $H_{\varphi}$ of (2.13) commutes with $V_{g}$ and $H_{\varphi}$ is diagonal with respect to the decomposition (2.22). To prove our proposition, we must consider two points, (i) the mass gap of $H_{\varphi}$ restricted to the vacuum sector and (ii) strict positivity of $H_{\varphi}$ in other sectors. Restricted to $A^{G}$, the $C^{*}$ dynamical systems $\gamma_{t}$ and $\gamma_{t}^{\tau}$ are mutually conjugate so $H_{\varphi}$ restricted to the vacuum sector has mass gap if and only if $H_{\psi}$ restricted to the vacuum sector has mass gap. Proposition 2.6 follows from the following lemma.

Lemma 2.8. If $\varphi$ is $q$ periodic and mixing, $H_{\varphi}$ cannot have mass gap on the vacuum sector if (2.15) is not valid for $H_{\varphi}$ in a non-vacuum sector.

Proof. Suppose that (2.15) is not valid for $H_{\varphi}$ in a sector $F_{\chi}$, namely, for any small $\varepsilon>0$

$$
\text { Spectrum } H_{\varphi} \text { on } F_{\chi} \cap(0, \varepsilon) \neq \phi .
$$

We now use the spectral theory of operator algebra (cf. Chap. 3 of [4]). By (2.23) we have an element $X_{\varepsilon}$ of $A$ which has spectrum (with respect to $\left.\gamma_{t}\right)$ in $(0, \varepsilon)$ and has the following properties:

$$
\begin{gathered}
\alpha_{g}\left(X_{\varepsilon}\right)=\chi(g) X_{\varepsilon}, \\
X_{\varepsilon} \Omega \neq 0 .
\end{gathered}
$$


The vector $X_{\varepsilon} \Omega$ is in the spectral subspace of $H_{\varphi}$ for $(0, \varepsilon)$. Let $m$ be the order of $\pi$ in the dual group $\hat{G}$ of $G$. Set

$$
I_{n_{1} n_{2} \ldots n_{m-1}}=\beta_{q n_{1}}\left(X_{\varepsilon}\right) \beta_{q n_{2}}\left(X_{\varepsilon}\right) \ldots \beta_{q n_{m-1}}\left(X_{\varepsilon}\right) X_{\varepsilon} \Omega \text {. }
$$

$I_{n_{1} n_{2} \ldots n_{m-1}}$ has spectrum in $(0, \varepsilon)$ and it belongs to the vacuum sector, so if for arbitrary small $\varepsilon$ ther exists $n_{1} n_{2} \ldots$ such that $I_{n_{1} n_{2} \ldots n_{m-1}}$ is nonzero, we get Lemma 2.8 .

To prove that (2.26) is not zero we use our assumption that $\varphi$ is factor and periodic. We first show that there exists $n$ such that

$$
I_{n}=\beta_{n q}\left(X_{\varepsilon}\right) X_{\varepsilon} \Omega \neq 0 .
$$

As our state is mixing we can find a subsequence of integers $n$ such that

$$
w-\lim _{n \rightarrow \infty} \frac{1}{2 n}\left(\sum_{m=-n}^{n} \beta_{m q}\left(X_{\varepsilon} * X_{\varepsilon}\right)\right)=\varphi\left(X_{\varepsilon} * X_{\varepsilon}\right) 1 .
$$

Then

$$
\begin{aligned}
\lim _{n \rightarrow \infty} \frac{1}{2 n}\left\{\sum_{m=-n}^{n}\left\|I_{m}\right\|^{2}\right\} & =\lim _{n \rightarrow \infty} \frac{1}{2 n}\left\{\sum_{m=-n}^{n}\left(X_{\varepsilon} \Omega, \beta_{m q}\left(X_{\varepsilon} * X_{\varepsilon}\right) X_{\varepsilon} \Omega\right)\right\} \\
& =\varphi\left(X_{\varepsilon} * X_{\varepsilon}\right)^{2}
\end{aligned}
$$

Hence by (2.25) there exists $n$ such that $I_{n}$ does not vanish.

We can repeat the same procedure to conclude that $I_{n_{1} n_{2} \ldots n_{m-1}}$ is nonzero for some $n$ 's.

The end of proof of Lemma 2.8 .

\section{String Order}

In this section, we consider the condition for purity of states after duality transformation. In this context, the notion of string order appears. The string order is introduced by den Nijs and Rommelse in the study of quantum antiferromagnets (cf. [10]). Kennedy and Tasaki argued that the string order characterizes the Haldane phase of the 1 dimensional antiferromagnetic Heisenberg model. We will see that the existence of string order is not sufficient for existence of mass gap. We need another condition.

We first recall the duality map of Kennedy and Tasaki:

$$
\begin{gathered}
V_{m}=\prod_{k=-m}^{m} Y_{k}, \\
Y_{k}=\left\{E_{k} \exp \left(i \pi S_{x}^{(k)}\right)+\left(1-E_{k}\right)\right\}, \\
E_{k}=\frac{1}{2}\left[1-\exp \left(i \pi \sum_{l=-m}^{k-1} S_{z}^{(l)}\right)\right], \\
\tau_{K T}(Q)=\lim _{m \rightarrow \infty} V_{m} Q V_{m}^{-1} .
\end{gathered}
$$


$V_{m}$ is a selfadjoint unitary. If the spin is an integer, we have

$$
\left[\exp \left(i \pi S_{x}\right), \exp \left(i \pi S_{z}\right)\right]=0
$$

thus $V_{m}$ is $G\left(=Z_{2} \times Z_{2}\right)$ invariant. It is easy to see for $m$ with $|j|<m$,

$$
\begin{gathered}
V_{m} S_{x}^{(\jmath)} V_{m}^{-1}=S_{x}^{(j)} \exp \left(i \pi \sum_{k=j+1}^{m} S_{x}^{(k)}\right) \\
V_{m} S_{z}^{(j)} V_{m}^{-1}=S_{z}^{(j)} \exp \left(i \pi \sum_{k=-m}^{j-1} S_{z}^{(k)}\right) \\
V_{m} S_{y}^{(\jmath)} V_{m}^{-1}=S_{y}^{(j)} \exp \left(i \pi \sum_{k=-m}^{j-1} S_{z}^{(k)}\right) \exp \left(i \pi \sum_{k=j+1}^{m} S_{x}^{(k)}\right) .
\end{gathered}
$$

As a consequence if $i<j$,

$$
\begin{gathered}
\tau_{K T}\left(S_{x}^{(i)} S_{x}^{(j)}\right)=\lim _{m \rightarrow \infty} V_{m} S_{x}^{(i)} S_{x}^{(j)} V_{m}^{-1}=S_{x}^{(i)} \exp \left(i \pi \sum_{k=i+1}^{j-1} S_{x}^{(k)}\right) S_{x}^{(j)} \\
\tau_{K T}\left(S_{z}^{(i)} S_{z}^{(j)}\right)=S_{z}^{(i)} \exp \left(i \pi \sum_{k=i+1}^{j-1} S_{z}^{(k)}\right) S_{z}^{(\jmath)} \\
\tau_{K T}\left(S_{y}^{(i)} S_{y}^{(j)}\right)=S_{y}^{(i)} \exp \left(i \pi \sum_{k=\imath+1}^{j-1} S_{z}^{(k)}\right) \exp \left(i \pi \sum_{k=i+1}^{j-1} S_{x}^{(k)}\right) S_{y}^{(\jmath)}
\end{gathered}
$$

Definition 3.1. Let $\varphi()$ be a translationally invariant state of $A . \varphi($ ) has the string order in the $x$ direction iff the following quantity does not vanish.

$$
\lim _{|i-j| \rightarrow \infty} \varphi\left(S_{x}^{(\imath)} \exp \left(i \pi \sum_{k=i+1}^{j-1} S_{x}^{(k)}\right) S_{x}^{(j)}\right) \neq 0
$$

The string order in the $y$ and $z$ direction is defined in the same manner.

As $\tau_{K T}^{2}=$ identity, $\varphi\left(\right.$ ) has string order iff the state $\psi()=\varphi \circ \tau_{K T} \circ E^{G}($ ) has the ordinary long range order. As $\tau_{K T}$ defined on $A^{G}$ cannot be extended to $A$ we have no relation between purity of states $\psi()$ and $\varphi()$. However it seems important to know criterions of purity of $\varphi()$ in terms of $\psi()$ for the study of deformation of AKLT Heisenberg models (see Sect. 5.3 of [5] and Sect. 4 of this paper).

For a state $\varphi()$ of $A$ we denote the restriction of $\varphi()$ to $A^{G}$ by $\varphi^{G}()$.

Let $\Theta_{\alpha}()(\alpha=x, y, z)$ be the automorphism of $A$ determined by

$$
\begin{gathered}
Q_{\alpha}(Q)=\exp \left(i \pi \sum_{k=-\infty}^{0} S_{\alpha}^{(k)}\right) Q \exp \left(-i \pi \sum_{k=-\infty}^{0} S_{\alpha}^{(k)}\right) Q \in A, \alpha=x, z, \\
\Theta_{y}(Q)=\Theta_{x} \circ \Theta_{z}(Q)
\end{gathered}
$$


Proposition 3.2. Let $\varphi()$ be a translationally and $G$ invariant state of $A$ and $\psi()$ be a state of $A$ defined by $\psi()=\varphi \circ \tau_{K T} \circ E^{G}()$. We assume (i) $\varphi^{G}\left(\right.$ ) (hence $\left.\psi^{G}()\right)$ is a pure state of $A^{G}$ and (ii) $\varphi$ () has the string order in $x y$ and $z$ directions. Then $\varphi()$ is pure iff all the states $\psi^{G}(),\left(\psi \circ \Theta_{\alpha}\right)^{G}()(\alpha=x, y, z)$ are mutually disjoint, namely, any two of these states yield mutually disjoint representations.

The proof of this result will be given at the end of this section.

Remark 3.3. We can also obtain criteria for the cases that the string order parameter of only one or two directions is nonvanishing or no string order in any direction. For example, we assume that $\varphi^{G}()$ (hence $\left.\psi^{G}()\right)$ is pure. Then when $\psi()$ is a pure state of $A$, the string order is absent in all the directions. In this circumstance, $\varphi()$ is pure iff all the states $\psi^{G}(),\left(\psi \circ \Theta_{\alpha}\right)^{G}()(\alpha=x, y, z)$ are unitarily equivalent. The conditions of other cases can be stated by equivalence of states. See the proof of Proposition 3.2.

Remark 3.4. In the same spirit as duality of Kennedy and Tasaki, we can introduce the notion of string order for Kramers Wannier duality for quantum Potts models. Here $G$ is $Z_{n}$ and recall definitions (1.4), (1.6) and (1.8). A $G$ invariant and translationally invariant state $\varphi($ ) (by the action of $\beta()$ ) has string order iff

$$
\lim _{i-j \rightarrow \infty} \varphi\left(V^{(j)} V^{(j+1)} \ldots V^{(i-1)} V^{(i)}\right) \neq 0
$$

In case of Kramers Wannier duality, we don't need the string order condition to describe of purity of state. More precisely, let $\Xi()$ be an automorphism determined by

$$
\Xi(Q)=\prod_{k=-\infty}^{0} V^{(k)} Q \prod_{k=-\infty}^{0} V^{(k)-1}
$$

Let $\varphi()$ be a $G$ invariant and translationally invariant state of $A$ and set $\psi()=$ $\varphi \circ \tau_{K W} \circ E^{G}()$. Suppose that $\varphi^{G}()$ (so $\left.\psi^{G}()\right)$ is a pure state of $A^{G}$. Then $\varphi($ ) is pure as a state of $A$ iff any two of $\psi^{G}$ and $\left(\psi \circ \Xi^{k}\right)^{G}(k=1,2, \ldots, n-1)$ are mutually disjoint.

The proof of Proposition 3.2 and the above remarks are based on the following lemma.

Lemma 3.5. Let $\varphi$ be a $G$ invariant state of $A, F_{\varphi}$ be the GNS representation space of $\varphi$ and $\Omega_{\varphi}$ be the GNS cyclic vector. Let $V_{g}$ be the unitary representation of $G$ implementing automorphisms $\beta_{g}$ ( $g$ in $G$ ).

$$
V_{g} Q V_{g}^{-1}=\beta_{g}(Q), \quad V_{g} \Omega_{g}=\Omega_{\varphi}
$$

We decompose $F_{\varphi}$ to the factor representations of $G$,

$$
F_{\varphi}=\sum_{\chi \in \hat{G}}^{\oplus} F_{\chi}
$$


On each $F_{\chi}, V_{g}$ acts as $\chi(g) 1$, where $\chi(g)$ is the character of $G$ (1 dimensional representation of $G)$. Let $\pi_{\chi}(\cdot)$ be the representation of $A^{G}$ restricted to $F_{\chi} \cdot \pi_{\chi}(\cdot)$ for the identity representation is referred to as vacuum representation (denoted by $\left.\pi_{1}(\cdot)\right)$.

(i) If $\pi_{1}(\cdot)$ is irreducible, all the other representations $\pi_{\chi}(\cdot)$ are irreducible.

(ii) The $G$ invariant state $\varphi$ is pure if and only if $\pi_{1}(\cdot)$ is irreducible and any two representations $\pi_{\chi}(\cdot), \pi_{\chi^{\prime}}(\cdot)$ appearing in (3.11) are mutually non-equivalent.

The proof of above lemma for the case of $G=Z_{2}$ is given in Lemma 4.1 and Lemma 8.1 of [1]. The proof can be applied in the present situation with a minor modification; we omit the details.

Proof of Proposition 3.2. We apply Lemma 3.5 with the group $Z_{2} \times Z_{2}$. The elements of $G$ are again denoted by $(\alpha, \beta)\left(\alpha, \beta=+\right.$ or - ). Let $G_{1}$ (respectively $G_{2}$ and $G_{3}$ ) be the subgroup $Z_{2}$ of $G$ generated by $(+,-)$ (respectively by $(-,+)$ and $(-,-)$ ). The existence of string order in the $x$ direction implies that $\psi()$ has the long range order in the $x$ direction.

$$
\lim _{|i-j| \rightarrow \infty} \psi\left(S_{x}^{(i)} S_{x}^{(j)}\right) \neq 0 .
$$

We have also long range order in $y$ and $z$ directions. We consider the GNS representation associated to $\psi()$.

Claim 1. Representations $\pi_{++}, \pi_{+-}, \pi_{-+}$and $\pi_{--}$of $A^{G}$ are all unitarily equivalent.

To see this claim, take the representation of $A^{G_{1}}$. By $G$ invariance of $\psi(),(3.12)$ tells us that $\psi()$ is not pure. By Lemma 3.5, the representations of $A^{G_{1}}$ restricted to $F_{++} \oplus F_{-+}$and $F_{--} \oplus F_{+-}$are unitarily equivalent. So the representations of $A^{G}$ restricted to $F_{++} \oplus F_{-+}$and $F_{--} \oplus F_{-+}$are unitarily equivalent. $\pi_{++}$is equivalent to either $\pi_{+-}$or $\pi_{--}$.

Suppose that $\pi_{++}$is equivalent to $\pi_{+-}$. Then we look at the string order in the $y$ direction and the same argument as above implies the equivalence of $\pi_{++} \oplus \pi_{+-}$and $\pi_{--} \oplus \pi_{-+}$. As a result, Claim 1 is valid.

If $\pi_{++}$is equivalent to $\pi_{--}$, we consider the string order in $z$ direction and we again have our claim.

We now turn to criteria for purity of $\varphi$. We denote the representation associated with $\varphi$ (respectively $\psi$ ) by $\pi^{\varphi}\left(\right.$ ) or $\pi_{\alpha \beta}^{\varphi}\left(\right.$ ) (respectively $\pi^{\psi}\left(\right.$ ) or $\left.\pi_{\alpha \beta}^{\psi}()\right)$. Due to Lemma 3.5 we have only to find conditions for disjointness of $\pi_{\alpha \beta}^{\varphi}()$. The representation $\pi_{++}^{\varphi}()$ of $A^{G}$ is disjoint from $\pi_{+-}^{\varphi}$ () if and only if representations of $A^{G}$ associated with $\varphi^{G}$ and $\varphi_{x}^{G}$ are disjoint provided $\varphi\left(S_{x}^{(j)^{2}}\right)$ is non-zero where

$$
\varphi_{x}^{G}(\cdot)=\frac{\varphi\left(S_{x}^{(j)} \cdot S_{x}^{(j)}\right)}{\varphi\left(S_{x}^{(j)^{2}}\right)} .
$$

Claim 2. There exists $j$ such that $\varphi\left(S_{x}^{(j)^{2}}\right)$ is non-zero. In fact, if $\varphi\left(S_{x}^{(j)^{2}}\right)$ vanishes for any $j, \varphi$ is the ground state of the trivial Hamiltonian $H$,

$$
H=\sum_{j \in Z} S_{x}^{(\jmath)^{2}}
$$


Equation (3.14) has a unique ground state which is the infinite tensor product of a vector state of $M_{3}(C)$. It is easy to show directly that this ground state has no string order.

Claim 3. Representations of $A^{G}$ associated with $\varphi^{G}$ are $\varphi_{x}^{G}$ are disjoint if and only if $\psi^{G}()$ and $\left(\psi \circ \Theta_{x}\right)^{G}()$ yield mutually disjoint representations of $A^{G}$.

To see this claim, we recall that $\tau_{K T}$ is an automorphism of $A^{G}$ so $\varphi^{G}$ and $\varphi_{x}^{G}$ are disjoint if and only if $\varphi^{G} \circ \tau_{K T}$ and $\varphi_{x}^{G} \circ \tau_{K T}$ are disjoint.

By definition, $\varphi^{G} \circ \tau_{K T}=\psi^{G}()$. Then

$$
\begin{aligned}
\varphi_{x}^{G} \circ \tau_{K T}(Q) \cdot \varphi\left(S_{x}^{(j)^{2}}\right) & =\varphi\left(S_{x}^{(j)} \tau_{K T}(Q) S_{x}^{(j)}\right)=\lim _{m \rightarrow \infty} \varphi\left(S_{x}^{(j)} V_{m} Q V_{m} S_{x}^{(j)}\right) \\
& =\lim _{m \rightarrow \infty} \varphi\left(V_{m}^{2} S_{x}^{(j)} V_{m} Q V_{m} S_{x}^{(j)} V_{m}^{2}\right)
\end{aligned}
$$

where $V_{m}$ is defined in (3.1). By use of (3.4) we have

$$
\begin{aligned}
(3.15) & =\lim _{m \rightarrow \infty} \varphi \circ \tau_{K T}\left(V_{m} S_{x}^{(j)} V_{m} Q V_{m} S_{x}^{(j)} V_{m}\right) \\
& =\psi\left(S_{x}^{(j)} e^{i \pi \sum_{k=0}^{j-1} S_{x}^{(k)}} \Theta_{x}(Q) S_{x}^{(\jmath)} e^{-i \pi \sum_{k=0}^{j-1} S_{x}^{(k)}} S_{x}^{(j)}\right) .
\end{aligned}
$$

By Claim 1 the states $\psi^{G}()$ and $\psi\left(S_{x}^{(j)} e^{i \pi \sum_{k=0}^{j-1} S_{x}^{(k)}} \cdot S_{x}^{(j)} e^{-i \pi \sum_{k=0}^{j-1} S_{x}^{(k)}} S_{x}^{(j)}\right)$ restricted to $A^{G}$ are equivalent. As a consequence we have Claim 3 .

We have the same type of results for $\psi^{G}(), \psi^{G} \circ \Theta_{y}()$ and $\psi^{G} \circ \Theta_{z}()$. Claim 3 and Lemma 3.5 lead us to Proposition 3.2.

End of proof for Proposition 3.2.

\section{An Application}

In this section, we consider the spin 1 model with the following Hamiltonian:

$$
H=\sum_{i} J_{i}\left[S_{i} \cdot S_{i+1}-\beta\left(S_{\imath} \cdot S_{i+1}\right)^{2}\right]
$$

with $J_{i}=1$ if $i$ is even and $0<J_{\imath}=\delta<1$ if $i$ is odd. In [5] it was proved that the ground state of this model has string order in the region where $|\beta+1 / 3|$ and $\delta$ are small. However the uniqueness of the periodic ground state and existence of mass gap were not proved for this model. Our results imply uniqueness of the periodic ground state and the existence of mass gap.

We have nothing to say about existence of the non-periodic ground state.

Theorem 4.1. There exists positive small constants $\varepsilon_{0}, \varepsilon_{1}, \varepsilon_{2}$ such that for $\delta$ and $\beta$ with $|\beta+1 / 3|<\varepsilon_{0}, \varepsilon_{1}<\delta<\varepsilon_{2}$ the Hamiltonian (4.1) has a unique periodic (period 2) ground state $\varphi() \cdot \varphi()$ has mass gap and exponentially fast decay of correlation.

The proof of above theorem is as follows. The model has $Z_{2} \times Z_{2}$ symmetry (by the action of (1.14)). Due to Lemma 2.1 and Remark 2.2 and polymer expansion, the 
$Z_{2} \times Z_{2}$ symmetric periodic ground state is unique so this state is $S U(2)$ invariant. We show that this $Z_{2} \times Z_{2}$ symmetric periodic ground state $\varphi()$ is mixing. In fact two point functions for $\varphi()$ decay exponentially fast. This was stated without proof in [5], so we sketch the proof here. After having established the mixing property, we can use Proposition 2.6 of (for $\psi()$ ) of this paper and [5]. Then Theorem 4.1 is a consequence of Proposition 2.6.

We now consider the exponential decay of the correlation. What is not standard in this computation is the following type of expectation value:

$$
\begin{aligned}
& \varphi\left(\alpha_{-m}\left(Q_{1}\right) S_{\alpha}^{(-m)} S_{\alpha}^{(m)} \alpha_{m}\left(Q_{2}\right)\right) \\
& \quad=\psi\left(\alpha_{-m}\left(\tilde{Q}_{1}\right) S_{\alpha}^{(-m)} e^{i \pi} \sum_{k=-m+1}^{m-1} S_{\alpha}^{(k)} S_{\alpha}^{(m)} \alpha_{m}\left(\tilde{Q}_{2}\right)\right)
\end{aligned}
$$

where $\alpha=x, y, z$ and $Q_{i} \in A^{Z_{2} \times Z_{2}}, \tilde{Q}_{i}=\tau_{K T}\left(Q_{i}\right)(i=1,2)$. As the state $\varphi()$ is $S U(2)$ invariant, we have only to consider the case $\alpha=z$. For simplicity of exposition we only consider

$$
\lim _{m \rightarrow \infty} \psi\left(S_{z}^{(-m)} e^{i \pi \sum_{k=-m+1}^{m-1} S_{z}^{(k)}} S_{z}^{(m)}\right)
$$

The general case can be considered in the same way.

We now look closely at the polymer expansion of [5]. We use the notation of [5] so $\omega_{\Lambda \beta}(\cdot)$ or $\omega_{\Lambda \beta}^{\mu}(\cdot)$ is a finite volume Gibbs state. As the observable $A_{m}$ is $Z_{2} \times Z_{2}$ invariant we don't have to consider pure ground states of $H_{\tau}$ and we have

$$
\lim _{\beta \rightarrow \infty} \lim _{\Lambda \rightarrow \infty} \omega_{\Lambda \beta}(\cdot)=\psi(\cdot)
$$

We set

$$
A_{m}=S_{z}^{(-m)} e^{i \pi \sum_{k=-m+1}^{m-1} S_{z}^{(k)}} S_{z}^{(m)}
$$

and we use the expansion

$$
\begin{aligned}
\omega_{\Lambda \beta}\left(A_{m}\right)= & \sum_{n \geq 0} \sum_{\chi\left(A_{m}\right), \chi_{1} \chi_{2} \ldots \chi_{n}} \\
& \times W_{A_{m}}\left(\chi\left(A_{m}\right)\right) W\left(\chi_{1}\right) W\left(\chi_{2}\right) \ldots W\left(\chi_{n}\right) \Psi_{c}\left(\chi\left(A_{m}\right), \chi_{1} \ldots \chi_{n}\right) .
\end{aligned}
$$

$W_{A}(\chi), W_{A}(C), \tilde{W}_{A}(C)$ and $\mu_{A}(C)$ are defined as in Sect. 5 of [5]. The support of weights $W_{A}(\chi), W_{A}(C), \tilde{W}_{A}(C)$ contain the support of $A$. For simplicity we set the shape of the block as the cube $(\tau=L)$.

In the present case, Lemmas 5.2-5.4 of [5] are replaced by

Lemma 5.2'. Given $\alpha>0$ we can choose $L, \beta_{0}$ large and $\varepsilon_{0} \eta$ small enough such that for $\beta>\beta_{0}$ and $|\varepsilon|<\varepsilon_{0}$,

$$
\left|W_{A_{m}}(\chi)\right|<C \exp \left(-\alpha\left(|\chi|-\frac{2 m}{L}\right)\right) \exp \left(-\eta \frac{2 m}{L}\right)
$$


where $\chi$ is the connected support of a polymer which intersects the support of $A_{m}$ and $C$ is a positive constant.

Lemma 5.3'. Given $\alpha>0$ we can choose $L, \beta_{0}$ large and $\varepsilon_{0} \eta$ small enough such that for $\beta>\beta_{0}$ and $|\varepsilon|<\varepsilon_{0}$,

$$
\left|\mu_{A_{m}}(C)\right|<\exp \left(-\alpha\left(|\chi|-\frac{2 m}{L}\right)\right) \exp \left(-\eta \frac{2 m}{L}\right)
$$

Lemma 5.4'. There exist constants $C$ and $K$ independent of $L, \tau, \beta$ such that

$$
\sum_{s(C)=\chi}\left|\tilde{W}_{A_{m}}(C)\right|<C \exp \left(K\left(|\chi|-\frac{2 m}{L}\right)\right)
$$

Lemma 5.2' and (4.5) implies decay of correlation. The crucial difference in the above estimate appears in (4.7) so we only give a proof of Lemma $5.3^{\prime}$ here.

Sketch of proof of Lemma 5.3'. We set $T=\exp \left(i \pi S_{z}\right) \otimes \exp \left(i \pi S_{z}\right)$ and let $\left\{e_{k, k=1,2, \ldots, 8}\right\}$ be the basis of appendix of [5]. Then

$$
\begin{aligned}
& T e_{1}=e_{2}, \quad T e_{2}=e_{1}, \quad T e_{3}=e_{4}, \quad T e_{4}=e_{3}, \\
& T e_{k}=e_{k} \quad \text { for } k=5,6,9, \quad T e_{k}=-e_{k} \quad \text { for } k=7,8 \text {. }
\end{aligned}
$$

Equation (4.9) may read that if a bond at the time $t=0$ is in a ground state of $V_{i j}$ the bond is another ground state in the subsequent time and the string of $\exp \left(i \pi S_{z}\right)$ has zero matrix elements between unperturbed ground states and excitation.

The key point of estimate (4.7) is the number $n$ of blocks $b_{1} b_{2} \ldots b_{n}$ in $\chi$ such that $\bar{b}_{1} \bar{b}_{2} \ldots \bar{b}_{n}$ do not have any block in common. Each block contributes the small factor $e^{-\alpha}$ as is the case of proof of Lemma 5.3 of [5].

In general, if a polymer $\chi$ has the boundary $\partial \chi$,

$$
n>\frac{|\chi|-|\partial \chi|}{25}+\frac{|\partial \chi|}{20}
$$

The denominator of the second term is smaller than that of the first term because the face of a block on the boundary of $\chi$ cannot touch any other blocks in $\chi$.

Now consider a possible support set $\chi$ for $\mu_{A}(C)$. Then

(i) At $x= \pm m$ with $t=0$ we have either exicted sites (for $V_{m, m+1}$ or $V_{-m-1-m}$ ) or $e_{j} \otimes e_{\imath}$ with $i \neq j, 1 \leq i, j \leq 4$, so the sites $x= \pm m$ with $t=0$ are connected with $\chi$.

(ii) Let $\nu$ be the intersection of $\chi$ and the segment $[-m, m]$ at $t=0$. On $\nu$ we get small factors like $e^{-\alpha|\nu|}$ because if a bond at $t=1 / N$ is not a ground state for $V_{i j}$ it is not either at $t=0$ due the string of $\exp \left(i \pi S_{z}\right)$ and (4.9).

(iii) If we take into account the curves in the boundary of $\chi$ which are connected to the segment $[-m, m]$ at $t=0$, we have $|\nu|+|\partial \chi| \geq 2 m$.

More precisely a connected part of the boundary which starts at the segment $[-m, m]$ at $t=0$ and which hits the $t=\beta$ wall or the end of the volume $\Lambda$ is longer 
than $2 m$ because both $\beta\left(>\beta_{0}\right)$ and $\Lambda$ are large, so a connected part of boundary with length less than $2 m$ must return to the segment $[-m, m]$ at $t=0$.

By (i), (ii) and (iii) (4.10) for a possible support set $\chi$ for $\mu_{A}(C)$ is

$$
\begin{aligned}
n>\frac{|\chi|-\left(\frac{2 m}{L}-|\nu|\right)}{25}+\left(\frac{1}{20}-\frac{1}{25}\right)|\partial \chi| & \geq \frac{|\chi|-\frac{2 m}{L}}{25}+\frac{1}{100}(|\nu|+|\partial \chi|) \\
& \geq \frac{|\chi|-\frac{2 m}{L}}{25}+\frac{1}{100} \cdot \frac{2 m}{L}
\end{aligned}
$$

Thus we have (4.7) with $\eta=\frac{\alpha}{50 L} m$.

The end of proof of Lemma 5.3'.

Acknowledgements. The author thanks Dr. Tom Kennedy and Dr. Hal Tasaki for showing him their preprint before publication.

\section{References}

1. Araki, H., Matsui, T.: Ground states of the $X Y$ model. Commun. Math. Phys. 101, 213-245 (1985)

2. Affleck, I., Lieb, E.H.: A proof of part of Haldane's conjecture on spin chains. Lett. Math. Phys. 12, 57 (1986)

3. Affleck, I., Lieb, E.H., Kennedy, T., Tasaki, H.: Valence bond ground states in isotropic quantum antiferromagnets. Commun. Math. Phys. 115, 477-528 (1988)

4. Brattelli, O., Robinson, D.: Operator algebras and quantum statistical mechanics (I) and (II). Berlin, Heidelberg, New York: Springer

5. Kennedy, T., Tasaki, H.: Hidden Symmetry breaking and the Haldane phase in $S=1$ quantum spin chains. Commun. Math. Phys. 147, 431-484 (1992)

6. Köberle, R., Marino, E.C.: Duality, mass spectrum and vacuum expectation values. Phys. Lett. B 126, 475-480 (1983)

7. Matsui, T.: Gibbs measure as quantum ground states. Commun. Math. Phys. 135, 79-90 (1990)

8. Matsui, T.: A Link between Classical and quantum Potts models. J. Stat. Phys. 59, 781-798 (1990)

9. Matsui, T.: Uniqueness of translationally invariant ground states in quantum spin systems. Commun. Math. Phys. 126, 453-467 (1990)

10. den Nijs, M.K., Rommelse, K.: Preroughening transitions in crystal surfaces and valence-bond phases in quantum spin chains. Phys. Rev. B 40, 4709 (1989) 
